\title{
Serum Serotonin Concentrations in Dogs with Degenerative Mitral Valve Disease
}

\author{
J.W. Arndt, C.A. Reynolds, G.E. Singletary, J.M. Connolly, R.J. Levy, and M.A. Oyama
}

Background: Increased serotonin (5HT) signaling has been implicated in valvular disease of humans and animals, including canine degenerative mitral valve disease (DMVD). High circulating 5HT concentration is a potential source of increased signaling, and serum 5HT concentrations have not been previously reported in dogs with DMVD.

Hypothesis: Dogs with DMVD and small breed dogs predisposed to DMVD have higher serum 5HT concentrations than large breed controls.

Animals: Fifty dogs affected with DMVD, 34 dogs predisposed to DMVD but without cardiac murmur or echocardiographic evidence of DMVD, and 36 healthy large breed control dogs.

Methods: Prospective analysis. Serum 5HT concentration was measured by an ELISA test.

Results: Median serum 5HT concentration was significantly higher in dogs with DMVD and in dogs predisposed to DMVD as compared with controls (DMVD, 765.5 ng/mL [interquartile range, 561.3-944.4]; predisposed, $774.9 \mathrm{ng} / \mathrm{mL}$ [528.3-1,026]; control, $509.8 \mathrm{ng} / \mathrm{mL}$ [320.8-708.8]; $P=.0001)$. Subgroup analysis of predisposed dogs indicated significantly higher serum 5HT concentrations in Cavalier King Charles Spaniel (CKCS) dogs than in other breeds (CKCS, 855.0 ng/mL [635.8-1,088]; non-CKCS, 554.2 ng/mL [380.6-648.4]; $P=.0023$ ). Age, platelet count, and platelet morphology were not correlated with 5HT concentration in any group.

Conclusions and Clinical Importance: Dogs with DMVD had significantly higher serum 5HT concentrations when compared with large breed control dogs. Healthy CKCS dogs had significantly higher serum 5HT concentrations than other healthy dogs predisposed to DMVD. Additional investigation into a possible role of 5HT in the pathogenesis of DMVD is warranted.

Key words: Canine myxomatous degeneration; Cardiac; Heart; Platelet function; Valvular disease.

D egenerative mitral valve disease (DMVD) is common in dogs, but relatively little is known about the molecular mechanisms that underlie its pathophysiology. Serotonin (5-hydroxytryptamine or 5HT)-related mechanisms are an important cause of human cardiac valve disease in the setting of carcinoid tumors, ${ }^{1}$ ergot alkaloid drug administration, ${ }^{2}$ and the dietary drug combination fenfluramine-phenteramine. ${ }^{3}$ In animal models, exogenous $5 \mathrm{HT}^{4,5}$ or blockade of $5 \mathrm{HT}$ clearance ${ }^{6}$ is associated with development of myxomatous-like degeneration. With respect to canine DMVD, increased transcription and translation of $5 \mathrm{HT}$ signaling components, including 5HT receptors, mitogen-activated protein kinases, tryptophan hydroxylase 1 , and related components of the transforming growth factor- $\beta$ (TGF- $\beta$ ) pathway, suggest a role of 5HT in the pathophysiology of this disease..$^{7-10, a, b}$ One potential source of increased 5HT signaling is circulating $5 \mathrm{HT}$. The objective of this study was to measure serum 5HT concentrations in dogs with naturally occur-

From the Department of Clinical Studies-Philadelphia, School of Veterinary Medicine, University of Pennsylvania, Philadelphia, PA (Arndt, Reynolds, Singletary, Oyama); and The Joseph Stokes Jr Research Institute, The Children's Hospital of Philadelphia, Philadelphia, PA (Connolly, Levy). All work on this project was performed at the School of Veterinary Medicine, University of Pennsylvania and The Children's Hospital of Philadelphia. This study has been presented in part as an abstract at the ACVIM Forum in San Antonio, TX, in June 2008.

Corresponding author: Mark A. Oyama, Department of Clinical Studies-Philadelphia, School of Veterinary Medicine, University of Pennsylvania, 3900 Delancey St., Philadelphia, PA 19104; e-mail: maoyama@vet.upenn.edu

Submitted April 30, 2009; Revised June 10, 2009; Accepted July 9, 2009.

Copyright (C) 2009 by the American College of Veterinary Internal Medicine

$10.1111 / j .1939-1676.2009 .0378 . x$
Abbreviations:
CKCS
DMVD
5HT
Cavalier King Charles Spaniels
TGF- $\beta$
serotonin
transforming growth factor- $\beta$

ring DMVD, small breed dogs predisposed to DMVD, and healthy large breed control dogs.

\section{Materials and Methods}

The study protocol was approved by the University of Pennsylvania Institutional Animal Care and Use Committee. Informed owner consent was obtained for all study patients. Three populations of dogs were prospectively recruited from client-owned dogs at the Matthew $\mathbf{J}$. Ryan Veterinary Hospital of the University of Pennsylvania. Entry criteria for the groups were as follows: (1) dogs with DMVD: body weight $<15 \mathrm{~kg}$, systolic murmur at the left cardiac apex, thickened mitral valve leaflets and mitral regurgitation detected on $2 \mathrm{D}$ and color flow Doppler echocardiography; (2) dogs predisposed to DMVD: body weight $<10 \mathrm{~kg}$, healthy with no evidence of heart murmur, normal 2D, M-mode, and color flow Doppler echocardiography; (3) large breed control group: body weight $>20 \mathrm{~kg},>3$ years of age, healthy with no evidence of heart murmur, normal 2D, M-mode, and color flow Doppler echocardiography. Patients receiving medications that potentially could alter serotonin concentrations (eg, fluoxetine) were excluded from the study.

Three milliliters of venous blood was drawn into plain red top tubes, centrifuged, and serum was extracted and transferred to clean glass tubes. Samples were stored at $-20^{\circ} \mathrm{C}$ and batch analyzed. Serum 5HT concentrations were measured by a commercially available ELISA. ${ }^{\mathrm{c}}$ The competitive ELISA acylates 5HT and utilizes a chemiluminescent peroxidase detection method. All samples were run in duplicate. For validation of the assay using canine samples, serum from 1 of the control dogs was spiked with increasing 
concentrations of 5HT. Linearity and parallelism were assessed using spiked canine samples and manufacturer controls for 5 different concentrations. Recovery was assessed as the percentage of recovered 5HT-spiked canine samples versus expected 5HT at 6 different concentrations.

In order to investigate the relationship between serum 5HT and platelets, $3 \mathrm{~mL}$ of blood for platelet count and morphology were drawn into EDTA-containing tubes from a subset of patients from each of the 3 groups. Platelet counts initially were performed by an automated system ${ }^{\mathrm{d}}$ and platelet morphology and verification or correction of platelet count were performed by visual inspection.

\section{Statistical Analysis}

Statistical analysis was performed by computer-based software. ${ }^{\text {e,f }}$ Descriptive statistics and median serum 5HT concentrations of the patient groups were compared by the Kruskal-Wallis test and Dunn's posthoc test. Serum 5HT between CKCS and nonCKCS breeds and between dogs with and without macroplatelets was compared by the Mann-Whitney test. Linearity of 5HT dilutions and correlation between platelet count and patient age with 5HT concentration were determined by linear regression and determination of the Pearson correlation coefficient. Parallelism of the 5HT assay was evaluated by comparing the slopes of 2 regression lines using a 2-tailed $P$ value with the null hypothesis that the lines would be parallel. A $P$ value of $<.05$ was considered significant.

\section{Results}

One hundred and twenty patients were enrolled. The control group consisted of 36 dogs including 20 females and 16 males with 15 mixed breed, 11 Great Danes, 3 Labrador Retrievers, 3 Weimaraner, 2 Golden Retrievers, and 1 each of American Pit Bull Terrier, and Bull Mastiff. The DMVD group consisted of 50 dogs including 24 females and 26 males with 37 CKCS, 3 mixed breed, 2 Yorkshire Terriers, 2 Pomeranians, 2 Maltese, and 1 each of Brittany Spaniel, Dachshund, Miniature Doberman Pinscher, and Rat Terrier Sixteen of 50 $(32 \%)$ dogs with DMVD were receiving cardiac medications at the time of enrollment. The medications and number of dogs receiving each drug were as follows: furosemide, 13; enalapril, 13; pimobendan, 6; aldactazide, 3; amlodipine, 2; benazepril, 2; digoxin, 2; and sildenafil,
1. The predisposed group consisted of 34 dogs including 23 females and 11 males with 25 CKCS, 2 mixed breed, and 2 Miniature Poodles, and 1 each of Cocker Spaniel, English Springer Spaniel, Jack Russell Terrier, Teacup Poodle, and Fox Terrier.

Dogs with DMVD were significantly older than control and predisposed dogs (DMVD, 8.5 years [5.5-11]; predisposed, 3.0 years [2.5-5.5]; control, 6.0 years [4.0 7.0]; $P<.0001)$. As expected, left ventricular internal dimension at end diastole and left atrial to aortic root dimension were significantly larger in dogs with DMVD than in predisposed and control dogs (Table 1).

The 5HT ELISA demonstrated good linearity $(r=0.96)$, parallelism $(P>.05)$, and recovery (mean recovery, $100.9 \%$; range, $89.4-107.5 \%$ ). Median serum 5HT concentrations in DMVD and predisposed dogs were significantly higher than in controls (DMVD, 765.5 ng/mL [561.3944.4]; predisposed, $774.9 \mathrm{ng} / \mathrm{mL}$ [528.3-1,026]; control, $509.8 \mathrm{ng} / \mathrm{mL}$ [320.8-708.8]; $P=.0001$ ) (Fig 1).

In the DMVD group, median 5HT concentration in CKCS was not significantly different than median 5HT concentration in non-CKCS (CKCS, $734.8 \mathrm{ng} / \mathrm{mL}$ [579.4-926.3]; non-CKCS, $821.5 \mathrm{mg} / \mathrm{mL}$ [458.8-1,017]; $P=1.0$; Fig 2A). However, within the predisposed group, the median 5HT concentration in CKCS was significantly higher than that of non-CKCS (CKCS, $855.0 \mathrm{ng} / \mathrm{mL}$ [635.8-1,088]; non-CKCS， $554.2 \mathrm{mg} / \mathrm{mL}$ [380.6-648.4]; $P=.0023$; Fig 2B). There was no significant correlation between age and 5HT concentration $\left(r^{2}=0.015, P=.17\right)$ in the study population. In dogs without DMVD (predisposed and control) there was a weak but significant negative correlation between age and serum 5HT concentration $\left(r^{2}=0.203, P<.0001\right.$; Fig 3$)$.

Platelet count and morphology were evaluated in 19 CKCS with DMVD, 7 predisposed CKCS, and 7 controls. There was no significant correlation between platelet count and serum 5HT in the entire study population $\left(r^{2}=0.0027, P=.78\right)$ nor in any individual group (DMVD, $r^{2}=0.0034, P=.81$; predisposed, $r^{2}=0.087$, $P=.52$; control, $\left.r^{2}=0.062, P=.58\right)$. Macroplatelets were present in $11 / 26(42 \%)$ of the CKCS, including $8 / 19$ $(42 \%)$ CKCS with DMVD, and 3/7 (43\%) predisposed

Table 1. Left ventricular internal and left atrial diameter indexed to aortic root dimension in dogs with DMVD, small breed dogs predisposed to DMVD, and large breed control dogs.

\begin{tabular}{lccrr}
\hline & DMVD & Predisposed & Control & $P$ \\
\hline LVIDd index & $2.14[1.92-2.56]^{\mathrm{a}}$ & $1.80[1.62-2.00]$ & $1.86[1.64-2.00]$ & $<.0001$ \\
LVIDs index & $1.25[1.14-1.43]$ & $1.12[0.99-1.27]^{\mathrm{b}}$ & $1.30[1.10-1.39]$ & .0042 \\
LA: Ao & $1.50[1.20-1.75]^{\mathrm{c}, \mathrm{d}}$ & $1.20[1.10-1.40]$ & $1.20[1.10-1.30]$ & $<.0001$ \\
FS\% & $41[34-47]$ & $37[35-40]$ & $31[28-37]^{\mathrm{eff}}$ & .0001 \\
\hline
\end{tabular}

${ }^{\mathrm{a}} P<.001$ versus predisposed and control.

${ }^{\mathrm{b}} P<.05$ versus DMVD and control.

${ }^{\mathrm{c}} P<.001$ versus control.

${ }^{\mathrm{d}} P<.01$ versus predisposed.

${ }^{\mathrm{e}} P<.001$ versus DMVD.

${ }^{\mathrm{f}} P<.05$ versus predisposed

Ao, aortic root dimension; FS, fractional shortening; LA, left atrial dimension; DMVD, degenerative mitral valve disease; LVIDd, left ventricular end-diastolic dimension; LVIDs left ventricular end-systolic dimension.

Superscripts denote significant differences between the 3 groups. 


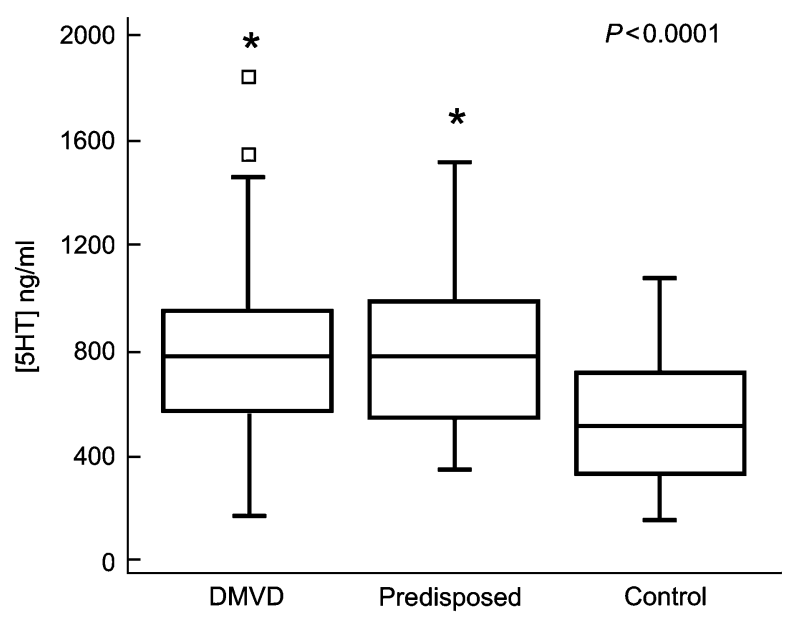

Fig 1. Box and whisker plot of serum 5HT concentration in dogs with degenerative mitral valve disease (DMVD), small breed dogs predisposed to DMVD, and healthy large breed controls. The line within the box represents the median value, the limits of the box represent the 25 th and 75 th percentile values and the whiskers represent the range. Outliers $>1.5$ interquartiles from the median are shown as squares. ${ }^{*} P<.001$ between groups.

CKCS. Macroplatelets were not detected in any of the control dogs. Median serum 5HT concentration was not significantly different in the CKCS with macroplatelets $(884 \mathrm{ng} / \mathrm{mL}[770.4-1,289])$ when compared with CKCS with normal platelet morphology $(789.7 \mathrm{ng} / \mathrm{mL}[595.5-$ $1,032], P=.32$ ).

\section{Discussion}

Increased 5HT signaling has been implicated in the development of valvular pathology in humans, ${ }^{1-3}$ animal models, ${ }^{4-6}$ and dogs with DMVD. ${ }^{7-9, a, b}$ In humans and animal models, many of the gross, histological, and molecular changes are similar to those seen in myxomatous DMVD of dogs. ${ }^{11}$ Our results indicate that dogs with naturally occurring DMVD as well as CKCS predisposed to DMVD have higher serum 5HT concentrations than do large breed controls. These results suggest a potential role for 5HT in the pathophysiology of DMVD in dogs, and particularly in the CKCS.

In humans, the role of 5HT in valve disease has been most studied in individuals with carcinoid tumors ${ }^{1,12}$ or in those receiving serotonergic medications such as fenfluramine or ergot alkaloids. ${ }^{2,3,13}$ In these instances, 5HT is believed to bind to 5HT receptors on the valve surface and activate downstream mitogenic pathways, resulting in differentiation of valvular interstitial cells, remodeling of the extracellular matrix, and abnormalities in collagen. ${ }^{9,14-18}$ 5HT-activated pathways include those involving extracellular regulated kinases, TGF- $\beta$, and protein kinase $C .{ }^{19-21}$ In humans, affected valves develop fibrous plaques, thickened leaflets and chordae tendineae, and glycosaminoglycan deposition in the extracellular matrix. ${ }^{1}$ The gross and histological changes seen with 5HT-mediated disease are similar but not identical to those seen in dogs with naturally occurring DMVD.
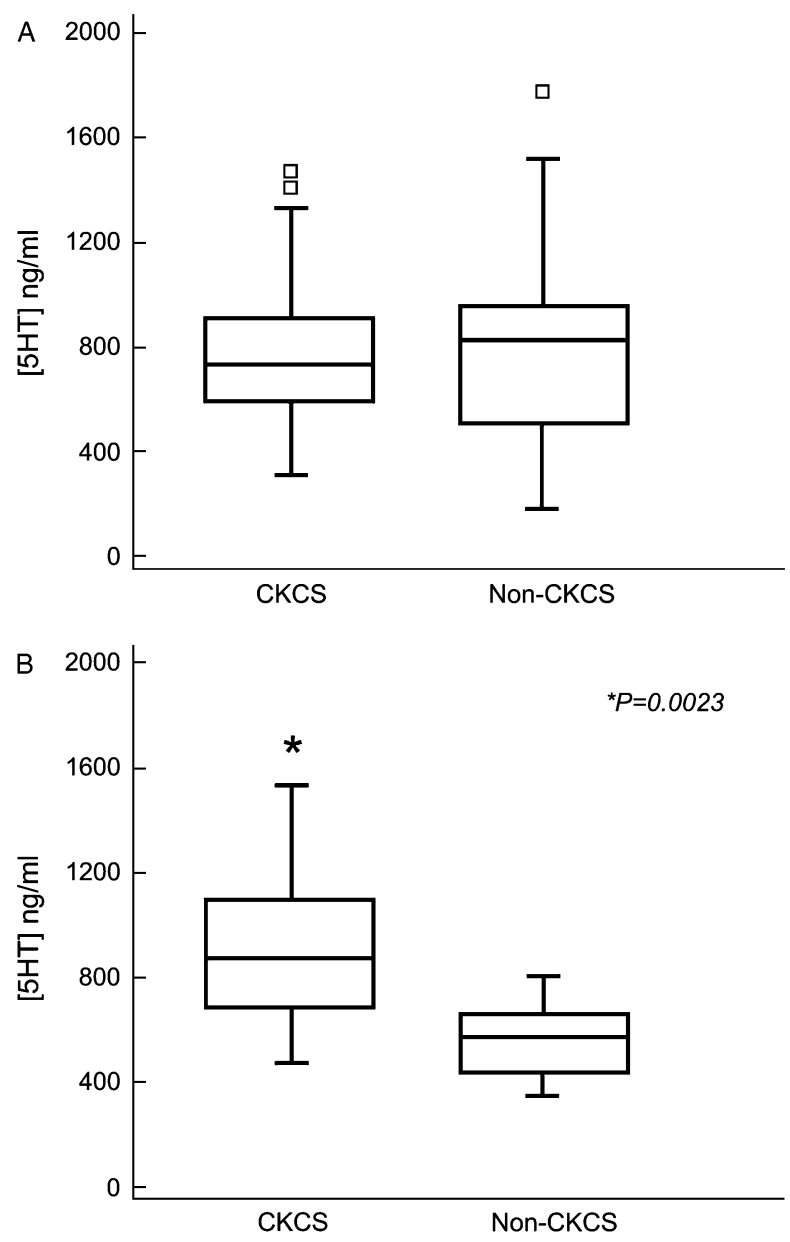

Fig 2. Box and whisker plot of serum $5 \mathrm{HT}$ concentration in Cavalier King Charles Spaniel (CKCS) dogs and non-CKCS dogs that were (A) affected with degenerative mitral valve disease (DMVD) or (B) predisposed to DMVD. CKCS dogs predisposed to DMVD had significantly greater serum 5HT than non-CKCS dogs. For both graphs, the line within the box represents the median value, the limits of the box represent the 25 th and 75 th percentile values and the whiskers represent the range. Outliers $>1.5$ interquartiles from the median are shown as squares.

Notably, dogs do not typically develop large fibrous plaques on the surface of the valve leaflets, but many of the changes, including proliferation and differentiation of valvular interstitial cells, deposition of myxoid ground substance, and neovascularization are similar between the 2 conditions. $^{22}$

A role for 5HT in canine DMVD previously has been suggested. Both the human ${ }^{23}$ and canine ${ }^{24}$ cardiovascular systems are rich in 5HT receptors, and expression of 5 HT receptors is increased in canine DMVD. ${ }^{7,9, a}$ Canine valvular interstitial cells exposed to exogenous 5HT activate mitogen-activated pathways and demonstrate increased expression of TGF- $\beta$ receptors and smooth muscle actin. ${ }^{8,9, a, b}$ Specifically, Disatian and Orton ${ }^{9}$ reported increased tryptophan hydroxylase in mitral tissues from dogs with DMVD, which suggests increased local production of 5HT, whereas our laboratory ${ }^{8}$ reported a dose-dependent increase in mitogenesis and 


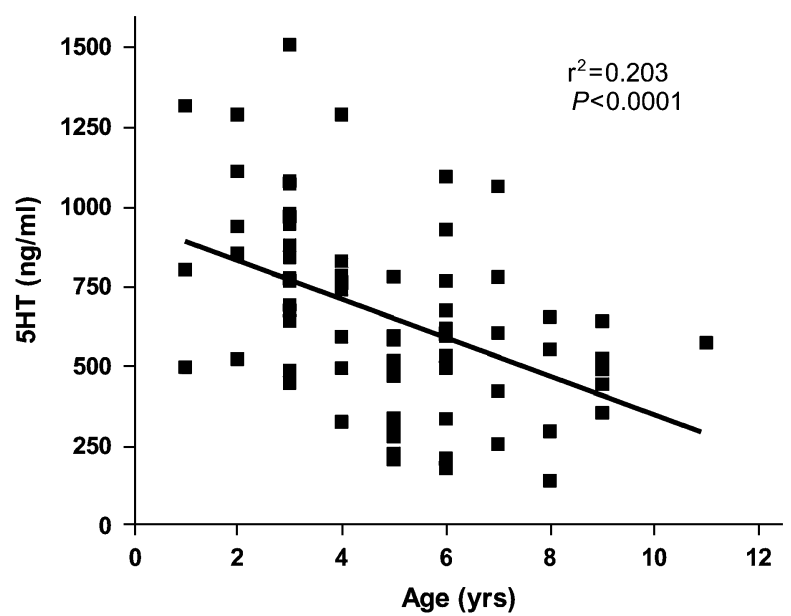

Fig 3. Linear regression of serum $5 \mathrm{HT}$ concentration versus age of healthy control dogs and dogs predisposed to degenerative mitral valve disease.

production of extracellular matrix and collagen when cultured canine mitral interstitial valve cells were incubated with $5 \mathrm{HT}$. These changes could be prevented by coincubation with ketanserin, a 5HT-2A receptor blocker or GR55562, a 5HT-1B receptor blocker, indicating that $5 \mathrm{HT}-2 \mathrm{~A}$ and $1 \mathrm{~B}$ receptor types are likely related to 5HT-induced mitogenesis and profileration. Some ${ }^{9}$ but not all studies ${ }^{\mathrm{b}}$ have shown that the serotonin transmembrane transporter (SERT), which clears and metabolizes 5HT, is simultaneously downregulated, further suggesting the potential for exaggerated autocrine 5HT signaling. SERT activity is affected by local production of nitric oxide by endothelial cells. ${ }^{25,26}$ Scanning electron microscopy demonstrates marked denudation of valvular endothelium from affected canine valve surfaces ${ }^{27}$ and this disruption also may contribute to local abnormalities in 5HT signaling. The end result of 5HT stimulation is differentiation of valvular interstitial cells into a more active myofibroblast phenotype. ${ }^{14}$ The hallmark of this activation is expression of smooth muscle actin. ${ }^{28,29}$ Thus, the results of previous studies indicate that 5HT signaling mechanisms are increased in mitral valve tissues of dogs with DMVD. Our results suggest that increased circulating concentrations of $5 \mathrm{HT}$ could be another potential source of increased 5HT signaling.

To the authors' knowledge, very few veterinary studies have reported serum $5 \mathrm{HT}$ concentration in dogs. Sako et $\mathrm{al}^{30}$ reported increased serum 5HT in a dog with a carcinoid tumor $(2,386 \mathrm{nmol} / \mathrm{L})$ as compared with 4 healthy dogs $(10-227 \mathrm{nmol} / \mathrm{L})$. Cakiroglu et $\mathrm{al},{ }^{31}$ studying behavioral aggression, reported a serum 5HT range of $12-32.5 \mathrm{ng} / \mathrm{mL}$ in 41 dogs. The manufacturer of the ELISA kit used in this study indicates a normal reference range in humans of $40-450 \mathrm{ng} / \mathrm{mL}$. By this standard, all 3 experimental groups in our study had median 5HT concentrations greater than the upper limit of the human reference range. To our knowledge, our study investigating serum $5 \mathrm{HT}$ is the largest to date, but additional studies are needed to confirm our findings and to determine if the modest increases in serum 5HT found in this study are sufficient to induce or propagate valve injury. The role of increased 5HT in healthy CKCS is particularly intriguing in that CKCS are highly predisposed to DMVD, and longitudinal studies are needed to determine if dogs with increased 5HT concentrations are more likely to develop DMVD. Interestingly, the inverse correlation between age and serum 5HT in healthy CKCS found in our study suggests that older dogs without DMVD tend to have lower 5HT.

The majority of circulating 5HT is contained in dense granules within platelets. Interestingly, platelets are attracted to cardiac injuries such as infarction, cardiomyopathy, and valve stenosis, and mediate activation of cardiac interstitial cells and fibroblasts by local 5HT release. ${ }^{32}$ In this environment, platelet-derived 5HT activates the TGF- $\beta$ pathway, increases extracellular matrix deposition, and activates matrix metalloproteinases. ${ }^{32}$ Thus, the hypothesis that canine valve injury may attract circulating platelets, which subsequently release $5 \mathrm{HT}$ and increase local 5HT signaling warrants further investigation. Also, approximately one-third of CKCS possess macrothrombocytes ${ }^{33}$ and several complex alterations in platelet and hemostatic function have been described in dogs with DMVD. ${ }^{33-38}$ In CKCS, the ultrastructure of the giant platelets appears normal by electron microscopy, ${ }^{33}$ but the actual 5HT content in the dense granules is unknown. Previous studies failed to find a correlation between the presence of macrothrombocytes and DMVD, ${ }^{37,39}$ which is consistent with our results indicating that serum 5HT was similarly increased in CKCS regardless of the presence of macrothrombocytes.

There are several important limitations to our study. Serum 5HT can be influenced by foods that are rich in tryptophan (eg, bananas, tomatoes, pineapples, walnuts), ${ }^{1}$ and we did not obtain samples from patients that were fasted or on standardized diets. The 3 patient groups were not matched with respect to age. However, the negative correlation between age and 5HT would tend to decrease, as opposed to increase, our ability to detect a difference between the DMVD and control groups. The presence of increased serum 5HT concentrations in dogs with DMVD does not necessarily indicate a causal relationship, and additional studies are needed to determine if circulating $5 \mathrm{HT}$ is a potential source of 5HT signaling in valve tissue, and if so, what combination of 5HT and valve conditions is needed to effect these changes. Importantly, serial examinations are required to determine the relationship between increased 5HT and development of DMVD in healthy CKCS and in other breeds. Our results only demonstrate that circulating (and therefore likely platelet) 5HT was increased in DMVD and CKCS, and evidence that platelets are attracted and adhere to degenerative mitral valves remains to be determined. Lastly, the gross and histological characteristics of hyperserotonergic disease and DMVD, while similar, are not identical, and additional studies to better characterize the role of $5 \mathrm{HT}$ in dogs with naturally occurring disease are needed. In conclusion, our results indicate that dogs with naturally occurring DMVD as well as asymptomatic CKCS have increased serum 5HT concentrations, which may be a source of increased 5HT 
signaling. The role of 5HT in the pathogenesis of DMVD warrants additional study.

\section{Footnotes}

${ }^{a}$ Oyama MA, Chittur SV, Rush JE, Keene BW. Expression of serotonin, transforming growth factor beta, and extracellular matrix signaling molecules in myxomatous mitral valve tissue. J Vet Intern Med 2008;33:716-7

${ }^{\mathrm{b}}$ Connolly JM, Oyama MA, Gorman RC, et al. Serotonin transporter blockade with dexfenfluoramine or fluoxetine increases serotonin-mediated ERK1/2 phosphorylation in heart valve interstitial cells: Implications for serotonin-related heart valve disease. Circulation 2006;114;III-300

${ }^{c}$ IB89527, Immuno Biological Laboratories Inc, Minneapolis, MN

${ }^{\mathrm{d}}$ Cell Dyn 3700, Abbott Diagnostics, Abbott Park, IL

${ }^{\text {e }}$ GraphPad Prism v4.0, GraphPad Software, La Jolla, CA

${ }^{\mathrm{f}}$ MedCalc v9.5.1.0, MedCalc Software, Mariakerke, Belgium

\section{Acknowledgments}

The authors thank Carolyn Michel CVT for her technical assistance and Dr Karen Jackson for evaluation of platelet counts and morphology.

\section{References}

1. Gustafsson BI, Hauso O, Drozdov I, et al. Carcinoid heart disease. Int J Cardiol 2008, epub ahead of print; doi:10.1016/j. ijcard.2008.02.019.

2. Horvath J, Fross RD, Kleiner-Fisman G, et al. Severe multivalvular heart disease: A new complication of the ergot derivative dopamine agonists. Mov Disord 2004;19:656-662.

3. Connolly HM, Crary JL, McGoon MD, et al. Valvular heart disease associated with fenfluramine-phentermine. N Engl J Med 1997;337:581-588.

4. Gustafsson BI, Tommeras K, Nordrum I, et al. Long-term serotonin administration induces heart valve disease in rats. Circulation 2005;111:1517-1522.

5. Elangbam CS, Job LE, Zadrozny LM, et al. 5-hydroxytryptamine (5HT)-induced valvulopathy: Compositional valvular alterations are associated with 5HT2B receptor and 5HT transporter transcript changes in Sprague-Dawley rats. Exp Toxicol Pathol 2008;60:253-262.

6. Mekontso-Dessap A, Brouri F, Pascal O, et al. Deficiency of the 5-hydroxytryptamine transporter gene leads to cardiac fibrosis and valvulopathy in mice. Circulation 2006;113:81-89.

7. Oyama MA, Chittur SV. Genomic expression patterns of mitral valve tissues from dogs with degenerative mitral valve disease. Am J Vet Res 2006;67:1307-1318.

8. Connolly JM, Bakay MA, Fulmer JT, et al. Fenfluramine disrupts the mitral valve interstitial cell response to serotonin. Am J Pathol 2009, doi:10.2353/ajpath.2009.081101.

9. Disatian S, Orton EC. Autocrine serotonin and transforming growth factor 31 signaling mediates spontaneous myxomatous mitral valve disease. J Heart Valve Dis 2009; 18:44-51.

10. Aupperle H, Marz I, Thielebein J, et al. Expression of transforming growth factor-beta1, -beta2 and -beta3 in normal and diseased canine mitral valves. J Comp Pathol 2008;139:97-107.

11. Donnelly KB. Cardiac valvular pathology: Comparative pathology and animal models of acquired cardiac valvular diseases. Toxicol Pathol 2008;36:204-217.
12. Fox DJ, Khattar RS. Carcinoid heart disease: Presentation, diagnosis, and management. Heart 2004;90:1224-1228.

13. Pritchett AM, Morrison JF, Edwards WD, et al. Valvular heart disease in patients taking pergolide. Mayo Clin Proc 2002;77: 1280-1286.

14. Rajamannan NM, Caplice N, Anthikad F, et al. Cell proliferation in carcinoid valve disease: A mechanism for serotonin effects. J Heart Valve Dis 2001;10:827-831.

15. Robiolio PA, Rigolin VH, Wilson JS, et al. Carcinoid heart disease. Correlation of high serotonin levels with valvular abnormalities detected by cardiac catheterization and echocardiography. Circulation 1995;92:790-795.

16. Waltenberger J, Lundin L, Oberg K, et al. Involvement of transforming growth factor-beta in the formation of fibrotic lesions in carcinoid heart disease. Am J Pathol 1993;142:71-78.

17. Cannistra LB, Gaasch WH. Appetite-suppressing drugs and valvular heart disease. Cardiol Rev 1999;7:356-361.

18. Rothman RB, Baumann MH. Appetite suppressants, cardiac valve disease and combination pharmacotherapy. Am J Ther 2009;16:354-364.

19. Jian B, Xu J, Connolly J, et al. Serotonin mechanisms in heart valve disease. I: Serotonin-induced up-regulation of transforming growth factor-beta1 via G-protein signal transduction in aortic valve interstitial cells. Am J Pathol 2002;161:2111-2121.

20. Xu J, Jian B, Chu R, et al. Serotonin mechanisms in heart valve disease. II: The 5-HT2 receptor and its signaling pathway in aortic valve interstitial cells. Am J Pathol 2002;161:2209-2218.

21. Grewal JS, Mukhin YV, Garnovskaya MN, et al. Serotonin 5-HT2A receptor induces TGF-betal expression in mesangial cells via ERK: Proliferative and fibrotic signals. Am J Physiol 1999;276: F922-F930.

22. Simula DV, Edwards WD, Tazelaar HD, et al. Surgical pathology of carcinoid heart disease: A study of 139 valves from 75 patients spanning 20 years. Mayo Clin Proc 2002;77:139-147.

23. Kaumann AJ, Levy FO. 5-hydroxytryptamine receptors in the human cardiovascular system. Pharmacol Ther 2006;111:674-706.

24. Bonaventure P, Nepomuceno D, Miller K, et al. Molecular and pharmacological characterization of serotonin 5-HT2A and 5-HT2B receptor subtypes in dog. Eur J Pharmacol 2005;513:181-192.

25. Bryan-Lluka LJ, Papacostas MH, Paczkowski FA, et al. Nitric oxide donors inhibit 5-hydroxytryptamine (5-HT) uptake by the human 5-HT transporter (SERT). Br J Pharmacol 2004;143:63-70.

26. Chanrion B, la Mannoury C, Bertaso F, et al. Physical interaction between the serotonin transporter and neuronal nitric oxide synthase underlies reciprocal modulation of their activity. Proc Natl Acad Sci USA 2007;104:8119-8124.

27. Corcoran BM, Black A, Anderson H, et al. Identification of surface morphologic changes in the mitral valve leaflets and chordae tendineae of dogs with myxomatous degeneration. Am J Vet Res 2004;65:198-206.

28. Cushing MC, Liao JT, Anseth KS. Activation of valvular interstitial cells is mediated by transforming growth factor-betal interactions with matrix molecules. Matrix Biol 2005;24:428-437.

29. Taylor PM, Batten P, Brand NJ, et al. The cardiac valve interstitial cell. Int J Biochem Cell Biol 2003;35:113-118.

30. Sako T, Uchida E, Okamoto M, et al. Immunohistochemical evaluation of a malignant intestinal carcinoid in a dog. Vet Pathol 2003;40:212-215.

31. Cakiroglu D, Meral Y, Sancak AA, et al. Relationship between the serum concentrations of serotonin and lipids and aggression in dogs. Vet Rec 2007;161:59-61.

32. Yabanoglu S, Akkiki M, Seguelas MH, et al. Platelet derived serotonin drives the activation of rat cardiac fibroblasts by 5 -HT2A receptors. J Mol Cell Cardiol 2009;46:518-525.

33. Cowan SM, Bartges JW, Gompf RE, et al. Giant platelet disorder in the Cavalier King Charles Spaniel. Exp Hematol 2004; 32:344-350. 
34. Tarnow I, Kristensen AT, Olsen LH, et al. Assessment of changes in hemostatic markers in Cavalier King Charles Spaniels with myxomatous mitral valve disease. Am J Vet Res 2004;65: $1644-1652$

35. Tanaka R, Yamane Y. Platelet aggregation in dogs with mitral valve regurgitation. Am J Vet Res 2000;61:1248-1251.

36. Tarnow I, Kristensen AT, Texel H, et al. Decreased platelet function in Cavalier King Charles Spaniels with mitral valve regurgitation. J Vet Intern Med 2003;17:680-686.
37. Olsen LH, Kristensen AT, Haggstrom J, et al. Increased platelet aggregation response in Cavalier King Charles Spaniels with mitral valve prolapse. J Vet Intern Med 2001;15:209-216.

38. Moesgaard SG, Sorensen TM, Sterup A, et al. Changes in platelet function in Dachshunds with early stages of myxomatous mitral valve disease. Res Vet Sci 2009;86:320-324.

39. Eksell P, Haggstrom J, Kvart C, et al. Thrombocytopenia in the Cavalier King Charles Spaniel. J Small Anim Pract 1994;35: $153-155$. 